\section{Teste de HIV solicitado e espontâneo: um estudo de base populacional com mulheres de uma cidade do Sul do Brasil}

\author{
Voluntary versus health professional-initiated HIV \\ testing: a population-based study in women in a \\ city in Southern Brazil
}

\section{Test de VIH solicitado y espontáneo: un estudio de base poblacional con mujeres de una ciudad del Sur de Brasil}

\section{Resumo}

Estudo transversal de base populacional foi realizado com o objetivo de descrever a prevalência, motivos e fatores associados para realização de teste para HIV entre mulheres de Pelotas, Sul do Brasil. Foram entrevistadas 1.222 mulheres. Foram estimados prevalência de teste (sim/não), teste solicitado e teste voluntário. A prevalência de teste foi de 66,1\% (IC95\%: 63,4-68,8), teste solicitado 52,4\% (IC95\%: 49,6-55,2) e teste espontâneo 13,6\% (IC95\%: 11,615,5). O principal motivo para realização de teste foi rastreamento pré-natal (52\%). Idade, idade da primeira relação e possuir filhos se mostraram associadas à realização de teste espontâneo e solicitado. Escores de comportamentos sexuais de risco, situação conjugal e uso de preservativo se mostraram associados apenas a teste solicitado e prática de sexo anal apenas a teste espontâneo. Os resultados indicam que testagem para HIV parece estar fortemente relacionada com a assistência pré-natal e que a percepção de risco por parte dos profissionais de saúde parece ser mais adequada do que a percepção da própria mulher.

HIV; Sorodiagnóstico da AIDS; Mulheres
Marilia Arndt Mesenburg 1

Fernando César Wehrmeister 1

Mariângela Freitas da Silveira 1

doi: 10.1590/0102-311X00074415

Correspondência

M. A. Mesenburg

Programa de Pós-graduação em Epidemiologia, Universidade Federal de Pelotas.

Rua Marechal Deodoro 1160, 3o andar, Pelotas, RS 96020-220, Brasil.

mariliaepi@gmail.com

1 Programa de Pós-graduação em Epidemiologia Universidade Federal de Pelotas, Pelotas, Brasil. 


\section{Introdução}

A epidemia de HIV/AIDS, desde seu início, afetou milhões de pessoas ao redor do mundo. Apesar dos esforços mundiais, que resultaram na queda global do número de novas infecções, alguns países, incluindo o Brasil, apresentam tendência de aumento na taxa geral de detecção do vírus 1. Entre 2004 e 2013, maior aumento foi observado entre homens jovens, porém se encontrou aumento também entre mulheres jovens e acima de 50 anos 2. Esse aumento na taxa de detecção aliado à elevação da expectativa de vida, proporcionada pela ampliação do acesso à terapia antirretroviral (TARV) e aos cuidados aos portadores do vírus, culmina com a ampliação do número de pessoas vivendo com HIV.

Estima-se que, em 2012, 20\% dos portadores do HIV no Brasil desconheciam seu status 2, o que aumenta a probabilidade de transmissão, já que comportamentos sexuais de alto risco, a principal forma de transmissão, são mais frequentes entre pessoas que desconhecem seu status soropositivo 3. O conhecimento do status sorológico pelo teste para detecção do HIV é a porta de entrada ao cuidado ao portador, que inclui a administração de TARV 2, determinante da redução da chance de transmissão do vírus durante o contato sexual, devido à supressão da carga viral 4 . No Brasil, o acesso à TARV é universal, portanto o maior desafio para a prevenção do HIV consiste na mudança comportamental em direção à adoção de comportamentos sexuais seguros e na consciência individual do status sorológico.

As mulheres, por questões biológicas, sociais e de relações desiguais de poder entre gêneros, são especialmente vulneráveis às doenças sexualmente transmissíveis (DST) e AIDS 5. Nos últimos anos, percebe-se uma diminuição da razão entre os sexos na epidemia de HIV/AIDS. Em termos mundiais, as mulheres já correspondem a mais da metade dos casos de HIV/AIDS no mundo ${ }^{6}$. No Brasil, a razão entre os sexos diminuiu ao longo dos anos, passando de 15,1 homens por mulher em $1980{ }^{7}$ para 1,6 em 2009 2. Apesar da estabilização da razão entre os sexos verificada desde então, mulheres jovens e acima dos 50 anos apresentam razão entre os sexos ainda menor por conta da tendência de aumento da taxa de detecção entre esses grupos 2 .

Diante de tal contexto, este estudo teve como objetivo descrever a prevalência e a motivação para realização de teste para detecção do HIV, e fatores demográficos, socioeconômicos e do comportamento sexual associados à realização de teste solicitado e teste espontâneo entre mulheres na cidade de Pelotas, Rio Grande do Sul, Brasil.

\section{Metodologia}

Este é um estudo transversal de base populacional realizado entre janeiro e julho de 2012, em Pelotas, uma cidade de aproximadamente 328 mil habitantes, localizada no extremo sul do Brasil. Trata-se de um recorte de um inquérito populacional maior realizado no contexto do Consórcio de Pesquisas do Programa de Pós-graduação em Epidemiologia da Universidade Federal de Pelotas 8, que investigou diversos temas relacionados à saúde e incluiu homens e mulheres de dez anos de idade ou mais. Este estudo inclui mulheres que se enquadravam nos seguintes critérios de elegibilidade: possuir entre 15 a 54 anos de idade, residir na zona urbana do município e declarar ter mantido relações sexuais alguma vez na vida, pois foi essa população que respondeu às questões sobre realização de teste para HIV. Foram excluídas do estudo mulheres institucionalizadas ou com alguma incapacidade que as impedisse de fornecer as informações desejadas.

O processo de amostragem foi realizado em múltiplos estágios, com base nos dados do Censo Demográfico de 2010, fornecidos pelo Instituto Brasileiro de Geografia e Estatística (IBGE; http:// www.ibge.gov.br). Os setores censitários, numerados pelo IBGE com base em sua localização geográfica, em formato de espiral, do centro para a periferia e em sentido horário, foram a unidade amostral primária. Primeiramente, os 495 setores censitários da cidade foram ordenados por sua numeração. A seguir, foram sorteados, sistematicamente, 130 setores. Considerando a ausência de dados sociodemográficos atualizados na época da realização do processo de amostragem, a estratégia supracitada foi a alternativa encontrada para assegurar a inclusão de setores de diversas regiões da cidade e com diferentes níveis socioeconômicos na amostra. Para corrigir defasagens em relação ao Censo 2010, em cada setor sorteado, foi realizada contagem do número de domicílios. A seguir, realizou-se sorteio sistemático de domicílios em cada setor, respeitando a probabilidade proporcional ao tamanho. Foram 
selecionados, em média, 12 domicílios por setor, permitindo assim a inclusão de 1.722 domicílios na amostra. Tendo em vista que o trabalho de campo foi realizado para investigação de múltiplos desfechos, o número de domicílios selecionados refere-se ao maior tamanho amostral necessário calculado. Todas as pessoas residentes nos domicílios selecionados foram convidadas a participar do estudo.

Os domicílios selecionados receberam a visita de uma entrevistadora treinada e com escolaridade mínima de Ensino Médio completo. Informações sobre realização de teste de HIV e práticas sexuais foram obtidas por meio de um questionário confidencial autoaplicado, que era entregue à mulher pela entrevistadora. Após o preenchimento do questionário, a entrevistada o colocava em um envelope, que era fechado com fita adesiva e depositado em uma urna lacrada, garantindo assim a confidencialidade das informações. Os dados provenientes desse questionário foram duplamente digitados no programa EpiData (Epidata Assoc., Odense, Dinamarca). As informações socioeconômicas, demográficas e sobre percepção de risco foram obtidas mediante questionários eletrônicos estruturados, aplicados em entrevistas face a face. As informações de ambos os questionários, eletrônico e autoaplicado, eram agregadas, com um número de identificação exclusivo para cada mulher, em um banco de dados único.

As variáveis realização e motivação para teste de HIV foram avaliadas pelas seguintes questões: "Você já fez algum teste para saber se tem o vírus da AIDS?" (sim/não) e "Qual foi o principal motivo pelo qual você fez o último teste?” (o patrão pediu/fiz pré-natal/doei sangue/achei que tinha algum risco/tive curiosidade/meu parceiro pediu/meu parceiro é HIV+/o médico pediu/exposição ocupacional/outro motivo, qual?).

Ao optar por marcar a opção de resposta “outro motivo, qual?", a própria entrevistada escrevia o que a motivou a realizar o teste. Todas as repostas provenientes dessa categoria foram analisadas e, quando possível, recodificadas nas categorias de reposta para motivo de realização do teste que constavam no questionário. Uma nova categoria de motivo para realização do teste foi criada, incluindo as repostas "fiz por precaução" ou "fiz motivada por campanha", as quais configuram atitudes de procura pela realização do teste, mas não se enquadravam em nenhuma outra opção de resposta do questionário. Ainda assim, três respostas foram mantidas na categoria outros por não se enquadrarem em nenhuma outra opção de resposta.

O desfecho realização de teste para HIV foi analisada de duas formas. Primeiramente, como variável dicotômica, com as opções de resposta sim e não, e, a seguir, como variável categórica nominal, contemplando três categorias: não realização de teste, realização de teste solicitado e realização de teste espontâneo, que foram definidas com base nas respostas da questão sobre motivo para realização de teste. Apesar da recomendação do Programa Conjunto das Nações Unidas sobre HIV/AIDS (UNAIDS) em excluir teste por doação de sangue, optou-se por considerar testes por esse motivo conforme realizado por França Junior et al. ${ }^{9}$, único estudo de base populacional brasileiro identificado até o momento. Tal procedimento foi adotado para permitir a comparabilidade com os resultados do presente estudo. Foram consideradas como tendo realizado teste solicitado as mulheres que assinalaram as seguintes opções: o patrão pediu, pré-natal, doou sangue, parceiro pediu e médico pediu. Foram consideradas como realizado teste espontâneo as mulheres que assinalaram respostas: percebeu risco, curiosidade, parceiro soropositivo, exposição ocupacional e precaução/campanha. As três respostas que foram mantidas na categoria outros, conforme mencionado anteriormente, foram recodificadas como missing.

Como variáveis independentes, foram utilizadas as seguintes informações: idade (anos completos, posteriormente categorizada para 15-19, 20-29, 30-39, 40-49 e 50-54), cor da pele (branca; não branca); renda per capita (em quintis), escolaridade (em anos completos de estudo, posteriormente categorizada), situação conjugal (com companheiro; sem companheiro), uso de preservativo na última relação (sim; não), múltiplos parceiros sexuais nos últimos três meses (sim; não - considerado não aquelas com um parceiro), prática de sexo anal na última relação (sim; não), primeira relação sexual precoce (sim; não - considerada como precoce a primeira relação sexual antes dos 18 anos); uso de álcool e/ ou droga pela mulher antes da última relação (sim; não), uso de álcool e/ou droga pelo parceiro antes da última relação (sim; não), percepção de risco de adquirir HIV ou qualquer outra DST, avaliada pela seguinte questão "Na sua opinião, qual a chance de você pegar AIDS ou qualquer outra doença pega pelo sexo?”, cujas opções de resposta muito possível, possível, pouco possível, quase impossível e impossível, doravante serão tratadas como muito elevada, elevada, moderada, baixa e muito baixa percepção de risco, ter tido pelo menos um filho biológico (sim; não), e escore de comportamentos 
sexuais de risco, construído pela atribuição de um ponto para cada comportamento sexual de risco referido pela entrevistada e posterior recategorização (nenhum comportamento; um comportamento; dois comportamentos; três ou mais comportamentos). Os comportamentos sexuais utilizados na construção do escore foram uso de preservativo na última relação, "múltiplos parceiros sexuais nos últimos três meses", prática de sexo anal na última relação, "primeira relação sexual precoce”; "uso de álcool e/ou droga pela mulher antes da última relação" e "uso de álcool e/ou droga pelo parceiro antes da última relação".

Primeiramente, foi descrita a distribuição de frequência das características dos indivíduos que compõem a amostra. A seguir, foi realizada a descrição da distribuição de frequência de realização de teste para HIV (independentemente de ter sido solicitado ou espontâneo) segundo as categorias das variáveis independentes. A mesma análise foi conduzida com a variável realização de teste para HIV em três categorias. Ainda foi feita a descrição da distribuição da frequência do motivo para realização de teste para HIV.

As análises subsequentes foram realizadas em duas etapas. Primeiramente, construiu-se um modelo utilizando como desfecho a variável dicotômica realização de teste para HIV. Tal modelo foi denominado "desfecho A". A seguir, construiu-se um segundo modelo, tendo como desfecho a variável "realização de teste para HIV em três categorias", categorizada em "nunca testada", "fez teste espontâneo" e "fez teste solicitado". Esse modelo foi denominado "desfecho B" e em todas as análises foi utilizada a categoria "nunca testada" como categoria de referência.

Nas análises, bruta e ajustada, para obtenção das razões de chances (RC) e respectivos intervalos de confiança (IC95\%), foi utilizada regressão logística para o desfecho A por ser dicotômico, e regressão logística multinomial para o desfecho B, por possuir três categorias. Valores de p foram obtidos pelo teste de Wald de heterogeneidade ou tendência, quando oportuno. Para os dois desfechos, utilizando as técnicas estatísticas supracitadas, foi conduzida análise multivariada, na qual foram incluídas as variáveis com valor de $\mathrm{p}<0,2$ na análise bivariada. Foi seguido modelo conceitual hierárquico baseado na literatura, que, no primeiro nível, encontram-se as variáveis socioeconômicas e demográficas (idade, cor da pele, renda, escolaridade e situação conjugal). No segundo nível, encontram-se as variáveis sobre comportamento sexual (uso de preservativo, uso de álcool/droga pela mulher, uso de álcool/ droga pelo parceiro, prática de sexo anal, múltiplos parceiros sexuais e primeira relação sexual precoce) e, no terceiro nível, as variáveis sobre ter tido filhos biológicos e percepção de risco. Para inclusão das variáveis na análise, foi usado o método de seleção backwards (retrógrada).

Para verificar o efeito independente do comportamento sexual de risco sobre a realização de teste para HIV, foi conduzida análise com uso de regressão logística e regressão multinomial, tendo como exposição o escore de comportamentos sexuais de risco. Nessa análise, o ajuste foi feito, primeiramente, para as variáveis do 1o nível hierárquico que apresentaram valor de $\mathrm{p}<0,20$ na análise bivariada e, a seguir, para a variável paridade.

Para avaliação da qualidade de ajuste do modelo, foram utilizados os seguintes parâmetros: critério de informação de Akaike (AIC) e critério de informação bayesiano (BIC), comparando o modelo utilizado com o modelo com todas as variáveis, no qual foram inseridas, simultaneamente, todas as variáveis.

Dentre todas as variáveis avaliadas por este estudo, a que apresentou maior efeito de delineamento amostral foi a não utilização de preservativo $(1,17)$ e a que apresentou maior percentual de valores ignorados foi primeira relação sexual precoce $(9,4 \%)$.

Para fins de controle de qualidade, $10 \%$ da amostra foram revisitadas, e a questão sobre percepção de risco foi reaplicada. O coeficiente de kappa, calculado para a concordância entre a resposta da questão sobre percepção de risco fornecida na entrevista e no controle de qualidade foi de 0,6.

As análises foram realizadas no programa Stata 12.0 (StataCorp LP, College Station, Estados Unidos), considerando o efeito de delineamento amostral. Este estudo atende à legislação que regulamenta a pesquisa com seres humanos e foi aprovado pelo Comitê de Ética em Pesquisa da Faculdade de Medicina da Universidade Federal de Pelotas, em 1o de dezembro de 2011 (77/11). Todas as participantes do estudo assinaram Termo de Consentimento Livre e Esclarecido. 


\section{Resultados}

Foram identificadas 1.465 mulheres elegíveis para o estudo, dentre as quais 134 não foram localizadas ou recusaram participar do estudo e cinco recusaram responder ao questionário confidencial, totalizando um percentual de perdas e recusas de 9\%. A amostra final foi composta por 1.222 mulheres que afirmaram já ter mantido relações sexuais.

\section{Análise descritiva}

Mais da metade das mulheres vivia com companheiro, 4/5 referiram cor da pele branca e cerca de dois terços já tinham tido pelo menos um filho biológico e não haviam utilizado preservativo na última relação. Aproximadamente $90 \%$ não apresentavam múltiplos parceiros sexuais nos três meses anteriores à entrevista, não relatou uso de bebida alcoólica antes da relação, seja esse uso próprio ou pelo parceiro e não relatou prática de sexo anal. A maioria manteve a primeira relação em idade precoce (58\%). A maior parte das entrevistadas possuía entre 40-49 anos de idade (28\%), entre 9-11 anos de estudo (34\%), encontrava-se no quintil superior de renda (23\%), apresentou percepção de risco muito baixa (37\%) e apresentou, no escore de comportamentos sexuais, um comportamento sexual de risco (39\%). A descrição das características da amostra, bem como a prevalência de realização de teste, teste solicitado e teste espontâneo estão descritas na Tabela 1 .

\section{Tabela 1}

Características descritivas da amostra e prevalência de realização de teste, teste solicitado e teste espontâneo para detecção do HIV, segundo características da amostra, entre mulheres de 15-54 anos. Pelotas, Rio Grande do Sul, Brasil, 2012.

\begin{tabular}{|c|c|c|c|c|}
\hline Variável & n (\%) & Teste (sim/não) (\%) & Teste solicitado (\%) & Teste espontâneo (\%) \\
\hline \multicolumn{5}{|l|}{ Idade (anos) } \\
\hline Até 19 & $114(9,3)$ & 28,3 & 21,2 & 7,1 \\
\hline $20-29$ & $315(25,7)$ & 67,9 & 54,3 & 13,5 \\
\hline $30-39$ & $300(24,6)$ & 85,8 & 71,2 & 14,6 \\
\hline $40-49$ & $342(28,0)$ & 66,0 & 51,2 & 14,8 \\
\hline $50-54$ & $151(12,4)$ & 52,1 & 37,7 & 14,4 \\
\hline \multicolumn{5}{|l|}{ Cor da pele } \\
\hline Branca & $981(80,3)$ & 65,4 & 51,8 & 13,7 \\
\hline Não branca & $241(19,7)$ & 68,8 & 55,3 & 13,5 \\
\hline \multicolumn{5}{|l|}{ Renda (em quintis) } \\
\hline 50 (inferior) & $213(18,0)$ & 64,8 & 47,1 & 17,6 \\
\hline 4 은 & $218(18,4)$ & 61,7 & 46,7 & 15,0 \\
\hline 3o & $239(20,2)$ & 62,8 & 48,7 & 14,1 \\
\hline 20 & $249(21,0)$ & 68,3 & 58,5 & 9,8 \\
\hline 1으 (superior) & $265(22,4)$ & 71,8 & 59,6 & 12,2 \\
\hline \multicolumn{5}{|c|}{ Escolaridade (anos completos) } \\
\hline Até 4 & $120(9,8)$ & 69,1 & 57,3 & 11,8 \\
\hline $5-8$ & $311(25,5)$ & 66,5 & 55,6 & 10,9 \\
\hline $9-11$ & $413(33,8)$ & 69,3 & 55,6 & 13,7 \\
\hline 12 ou mais & $378(30,9)$ & 61,4 & 45,0 & 16,4 \\
\hline \multicolumn{5}{|l|}{ Situação conjugal } \\
\hline Sem companheiro & $527(43,1)$ & 58,7 & 42,3 & 16,4 \\
\hline Com companheiro & $695(56,9)$ & 71,6 & 60,1 & 11,6 \\
\hline \multicolumn{5}{|l|}{ Uso de preservativo } \\
\hline Sim & $422(35,3)$ & 59,1 & 45,1 & 14,0 \\
\hline Não & $773(64,7)$ & 70,0 & 56,6 & 13,4 \\
\hline
\end{tabular}

(continuação) 
Tabela 1 (continuação)

\begin{tabular}{|c|c|c|c|c|}
\hline Variável & n (\%) & Teste (sim/não) (\%) & Teste solicitado (\%) & Teste espontâneo (\%) \\
\hline \multicolumn{5}{|c|}{ Múltiplos parceiros sexuais } \\
\hline Até 1 & $1.073(95,0)$ & 66,7 & 53,2 & 13,5 \\
\hline 2 ou mais & $56(5,0)$ & 62,5 & 48,2 & 14,3 \\
\hline \multicolumn{5}{|c|}{ Prática de sexo anal } \\
\hline Não & $1.144(95,6)$ & 65,1 & 51,8 & 13,3 \\
\hline Sim & $53(4,4)$ & 84,9 & 64,2 & 20,8 \\
\hline \multicolumn{5}{|c|}{ Primeira relação sexual precoce } \\
\hline Não & $460(41,6)$ & 62,0 & 48,0 & 14,0 \\
\hline Sim & $647(58,4)$ & 67,8 & 54,6 & 13,2 \\
\hline \multicolumn{5}{|c|}{ Uso de álcool ou droga pela mulher } \\
\hline Não & $1.130(93,8)$ & 65,8 & 52,3 & 13,5 \\
\hline Sim & $74(6,2)$ & 69,9 & 56,2 & 13,7 \\
\hline \multicolumn{5}{|c|}{$\begin{array}{l}\text { Uso de álcool e/ou droga pelo } \\
\text { parceiro }\end{array}$} \\
\hline Não & $1.054(88,6)$ & 65,0 & 52,0 & 13,0 \\
\hline Sim & $136(11,4)$ & 73,5 & 55,2 & 18,4 \\
\hline \multicolumn{5}{|c|}{ Percepção de risco a HIV/DST } \\
\hline Muito baixa & $455(37,3)$ & 63,0 & 52,6 & 10,4 \\
\hline Baixa & $320(26,2)$ & 65,5 & 51,9 & 13,6 \\
\hline Moderada & $247(20,3)$ & 68,2 & 52,1 & 16,1 \\
\hline Elevada & $142(11,6)$ & 69,3 & 49,3 & 20,0 \\
\hline Muito elevada & $55(4,6)$ & 75,0 & 62,5 & 12,5 \\
\hline \multicolumn{5}{|c|}{ Escore de comportamentos sexuais } \\
\hline Nenhum & $131(12,7)$ & 57,3 & 43,5 & 13,7 \\
\hline 1 & $404(39,2)$ & 61,5 & 48,4 & 13,2 \\
\hline 2 & $380(36,8)$ & 69,4 & 55,9 & 13,6 \\
\hline 3 ou mais & $117(11,3)$ & 75,2 & 59,0 & 16,2 \\
\hline \multirow{2}{*}{\multicolumn{5}{|c|}{$\begin{array}{l}\text { Ter tido pelo menos um filho } \\
\text { biológico }\end{array}$}} \\
\hline & & & & \\
\hline Não & $384(31,5)$ & 42,9 & 27,1 & 15,8 \\
\hline Sim & $836(68,5)$ & 76,9 & 64,4 & 12,5 \\
\hline
\end{tabular}

A realização de teste de HIV, independentemente se solicitado ou espontâneo, foi referida por 66,1\% (IC95\%: 63,4-68,8) das mulheres. Maiores prevalências foram encontradas entre mulheres com 30-39 anos (86\%), não brancas (69\%), pertencentes ao primeiro quintil de renda (72\%), com 9-11 anos de estudo (69\%), com companheiro (72\%), que não utilizaram preservativo na última relação (70\%), não referiram múltiplos parceiros nos três meses anteriores à entrevista (67\%), praticaram sexo anal na última relação sexual (85\%), mantiveram a primeira relação em idade precoce (68\%), utilizaram álcool antes da última relação (70\%), cujo parceiro utilizou álcool antes da última relação (74\%), com percepção de risco muito elevada (75\%), que tiveram pelo menos um filho biológico (77\%) e relataram três ou mais comportamentos sexuais de risco (75\%).

A prevalência de realização de teste solicitado foi de 52,4\% (IC95\%: 49,6-55,2), maior entre mulheres com 30-39 anos (71\%), não brancas (55\%), pertencentes ao quintil superior de renda (60\%), com 9-11 anos de estudo (57\%), com companheiro (60\%), que não utilizaram preservativo na última relação (57\%), que não relataram múltiplos parceiros (53\%), que praticaram sexo anal na última relação sexual (64\%), que mantiveram a primeira relação em idade precoce (55\%), que utilizaram álcool antes da última relação (56\%), cujo parceiro utilizou álcool antes da última relação (55\%), com percepção de risco muito elevada (61\%), que tiveram pelo menos um filho biológico (64\%) e relataram três ou mais comportamentos sexuais de risco (59\%). 
A prevalência de teste espontâneo foi de 13,6\% (IC95\%: 11,6-15,5). Menor prevalência foi verificada entre aquelas com 19 anos ou menos (7\%), mantendo-se em torno de $14 \%$ nas demais faixas etárias. Maior prevalência foi constatada entre aquelas pertencentes ao quintil inferior de renda (18\%), com 12 ou mais anos de escolaridade (16\%), sem companheiro (16\%), que referiram prática de sexo anal durante a última relação (21\%), cujo parceiro utilizou álcool antes da última relação (18\%), com percepção de risco elevada (20\%), que não tiveram nenhum filho biológico (16\%) e relataram três ou mais comportamentos sexuais de risco (16\%). Os percentuais de teste espontâneo se mantiveram em cerca de 13\%, independentemente de cor da pele, uso de preservativo, múltiplos parceiros sexuais, primeira relação sexual precoce e uso de álcool e/ou droga pela mulher.

Com relação ao motivo para realização de teste para HIV, os mais citados foram realização de prénatal (52\%), doação de sangue (12\%), curiosidade (11\%) e solicitação médica (11\%). A distribuição de frequência dos motivos para realização de teste para HIV é apresentada na Figura 1.

Os resultados das análises seguintes, apresentados nas Tabelas 2 e 3 , serão descritos separadamente para o desfecho A e para o desfecho B.

\section{- Análise do desfecho A}

A análise bruta identificou associação entre teste para HIV e as variáveis idade, situação conjugal, uso de preservativo, prática de sexo anal e parição. $\mathrm{Na}$ análise ajustada, a associação com a variável uso de preservativo deixou de apresentar significância, enquanto a variável primeira relação precoce manteve-se associada. As variáveis renda, escolaridade, cor da pele, e múltiplos parceiros nos últimos três meses não apresentaram associação com o desfecho. A idade da mulher mostrou-se fortemente associada à realização de teste. Em comparação à faixa etária de até 19 anos, a chance de realização de teste apresentou um aumento de $456 \%, 1.315 \%, 339 \%$ e $144 \%$ entre aquelas com 20-29 anos, 30-39 anos, 40-49 anos e 50-54 anos, respectivamente. Mulheres que declararam viver com companheiro

Figura 1

Distribuição de frequência do principal motivo para realização do último teste para detecção do HIV entre mulheres de 15-54 anos.

Pelotas, Rio Grande do Sul, Brasil, 2012.

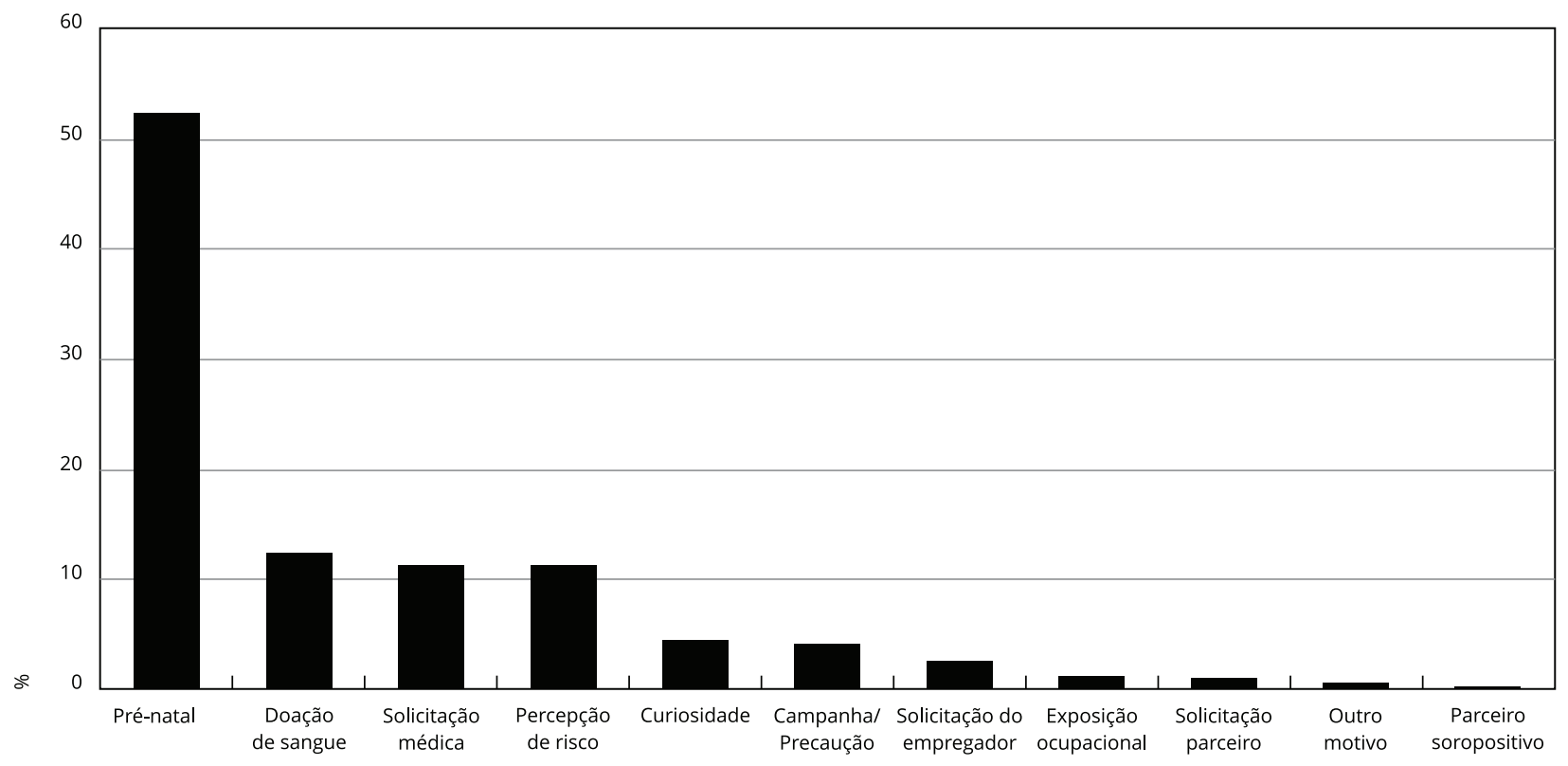


Tabela 2

Razões de chance bruta e ajustada de realização de teste, teste solicitado e teste espontâneo para detecção do HIV, segundo características da amostra, entre mulheres de 15-54 anos. Pelotas, Rio Grande do Sul, Brasil, 2012.

\begin{tabular}{|c|c|c|c|c|c|c|}
\hline \multirow[t]{4}{*}{ Variável } & \multirow{2}{*}{\multicolumn{2}{|c|}{$\begin{array}{c}\text { Modelo A * } \\
\text { Teste (sim/não) }\end{array}$}} & \multicolumn{4}{|c|}{ Modelo B ** } \\
\hline & & & \multirow{2}{*}{\multicolumn{2}{|c|}{$\begin{array}{l}\text { Teste solicitado } \\
\text { RC (IC95\%) }\end{array}$}} & \multirow{2}{*}{\multicolumn{2}{|c|}{$\begin{array}{l}\text { Teste espontâneo } \\
\text { RC (IC95\%) }\end{array}$}} \\
\hline & \multicolumn{2}{|c|}{ RC (IC95\%) } & & & & \\
\hline & Bruta & Ajustada *** & \multicolumn{2}{|r|}{ Ajustada *** } & Bruta & Ajustada *** \\
\hline Idade (anos) & $<0,001 \#$ & $<0,001 \#$ & $<0,001 \#$ & $<0,001 \#$ & & \\
\hline Até 19 & 1,00 & 1,00 & 1,00 & 1,00 & 1,00 & 1,00 \\
\hline $20-29$ & $5,34(3,19-8,9)$ & $5,56(3,32-9,33)$ & $5,7(3,34-9,71)$ & $6,14(3,47-10,88)$ & $4,25(1,90-9,48)$ & $4,43(1,96-10,04)$ \\
\hline $30-39$ & $15,24(8,3-27,74)$ & $14,15(7,75-25,84)$ & $16,87(9,19-30,96)$ & $15,12(7,98-28,65)$ & $10,36(4,39-24,46)$ & $10,70(4,40-26,04)$ \\
\hline $40-49$ & $4,90(2,87-8,37)$ & $4,39(2,53-7,61)$ & $5,07(2,91-8,84)$ & $4,46(2,47-8,06)$ & $4,39(1,94-9,88)$ & $4,50(1,98-10,19)$ \\
\hline $50-54$ & $2,74(1,53-4,28)$ & $2,44(1,34-4,43)$ & $2,65(1,42-4,92)$ & $2,66(1,37-5,18)$ & $3,03(1,37-6,73)$ & $3,15(1,37-7,11)$ \\
\hline Cor da pele & $0,371 \#$ & & $0,655 \#$ & & & \\
\hline Branca & 1,00 & - & 1,00 & - & 1,00 & - \\
\hline Não branca & $1,16(0,83-1,62)$ & - & $1,18(0,82-1,73)$ & - & $1,09(0,67-1,77)$ & - \\
\hline Renda (em quintis) & $0,102 \#$ & $0,148 \#$ & $0,013 \#$ & $0,007 \#$ & & \\
\hline $1 \underline{0}$ & 1,00 & 1,00 & 1,00 & 1,00 & 1,00 & 1,00 \\
\hline 20 & $0,87(0,59-1,28)$ & $0,78(0,52-1,16)$ & $0,91(0,61-1,35)$ & $0,92(0,60-1,40)$ & $0,78(0,43-1,39)$ & $0,79(0,4-1,42)$ \\
\hline 3o & $0,91(0,60-1,38)$ & $0,74(0,46-1,17)$ & $0,97(0,64-1,48)$ & $0,97(0,63-1,48)$ & $0,75(0,41-1,39)$ & $0,76(0,41-1,42)$ \\
\hline 40 & $1,17(0,77-1,76)$ & $0,99(0,61-1,62)$ & $1,37(0,90-2,09)$ & $1,48(0,95-2,31)$ & $0,61(0,33-1,14)$ & $0,67(0,36-1,25)$ \\
\hline 50 & $1,38(0,94-2,02)$ & $1,25(0,77-2,05)$ & $1,57(1,06-2,34)$ & $1,79(1,17-2,73)$ & $0,86(0,46-1,58)$ & $0,96(0,51-1,80)$ \\
\hline $\begin{array}{l}\text { Escolaridade (anos } \\
\text { completos) }\end{array}$ & $0,101 \#$ & $0,019 \#$ & $0,020 \#$ & $0,205 \#$ & & \\
\hline Até 4 & 1,00 & 1,00 & 1,00 & 1,00 & 1,00 & 1,00 \\
\hline $5-8$ & $0,88(0,52-1,49)$ & $0,84(0,48-1,49)$ & $0,89(0,51-1,53)$ & $0,92(0,51-1,65)$ & $0,84(0,41-1,73)$ & $0,92(0,43-1,99)$ \\
\hline $9-11$ & $1,00(0,61-1,64)$ & $0,99(0,58-1,68)$ & $0,97(0,58-1,63)$ & $1,18(0,66-2,12)$ & $1,16(0,62-2,15)$ & $1,24(0,63-2,42)$ \\
\hline 12 ou mais & $0,71(0,43-1,15)$ & $0,62(0,37-1,04)$ & $0,62(0,37-1,05)$ & $0,69(0,37-1,28)$ & $1,10(0,57-2,13)$ & $0,83(0,37-1,86)$ \\
\hline Situação conjugal & $<0,001 \#$ & $0,010 \#$ & $<0,001 \#$ & $<0,001 \#$ & & \\
\hline Sem companheiro & 1,00 & 1,00 & 1,00 & 1,00 & 1,00 & 1,00 \\
\hline Com companheiro & $1,77(1,42-2,22)$ & $1,39(1,08-1,80)$ & $2,06(1,64-2,60)$ & $1,70(1,29-2,24)$ & $1,02(0,70-1,50)$ & $0,79(0,53-1,17)$ \\
\hline Uso de preservativo & $<0,001 \#$ & $0,064 \#$ & $<0,001 \#$ & $0,093 \#$ & & \\
\hline Sim & 1,00 & 1,00 & 1,00 & 1,00 & 1,00 & 1,00 \\
\hline Não & $1,61(1,27-2,03)$ & $1,31(0,98-1,76)$ & $1,70(1,34-2,16)$ & $1,39(1,02-1,90)$ & $1,30(0,89-1,90)$ & $1,10(0,71-1,71)$ \\
\hline $\begin{array}{l}\text { Múltiplos parceiros } \\
\text { sexuais }\end{array}$ & $0,484 \#$ & & $0,748 \#$ & & & \\
\hline Até 1 & 1,00 & - & 1,00 & - & 1,00 & - \\
\hline 2 ou mais & $0,83(0,49-1,39)$ & & $0,80(0,45-1,41)$ & - & $0,93(0,40-2,16)$ & - \\
\hline Prática de sexo anal & $0,007 \#$ & $0,034 \#$ & $0,020 \#$ & $0,057 \#$ & & \\
\hline Não & 1,00 & 1,00 & 1,00 & 1,00 & 1,00 & 1,00 \\
\hline $\operatorname{Sim}$ & $3,37(1,51-7,53)$ & $2,50(1,07-5,83)$ & $2,86(1,22-6,67)$ & $2,38(0,92-6,09)$ & $3,61(1,40-9,32)$ & $3,57(1,26-10,13)$ \\
\hline $\begin{array}{l}\text { Primeira relação } \\
\text { sexual precoce }\end{array}$ & $0,052 \#$ & $0,021 \#$ & $0,123 \#$ & 0,019\# & & \\
\hline Não & 1,00 & 1,00 & 1,00 & 1,00 & 1,00 & 1,00 \\
\hline Sim & $1,29(0,99-1,66)$ & $1,40(1,05-1,87)$ & $1,34(1,01-1,77)$ & $1,51(1,09-2,08)$ & $1,11(0,78-1,58)$ & $1,57(1,04-2,36)$ \\
\hline $\begin{array}{l}\text { Uso de álcool ou } \\
\text { droga pela mulher }\end{array}$ & $0,472 \#$ & & $0,771 \#$ & & & \\
\hline Não & 1,00 & - & 1,00 & - & 1,00 & - \\
\hline Sim & $1,20(0,72-2,00)$ & - & $1,21(0,70-1,33)$ & - & $1,14(0,56-2,34)$ & - \\
\hline $\begin{array}{l}\text { Uso de álcool e/ou } \\
\text { droga pelo parceiro }\end{array}$ & $0,055 \#$ & $0,256 \#$ & $0,096 \#$ & $0,361 \#$ & & \\
\hline Não & 1,00 & 1,00 & 1,00 & 1,00 & 1,00 & 1,00 \\
\hline Sim & 1,49 $(0,99-2,26)$ & $1,31(0,81-2,09)$ & $1,40(0,91-2,15)$ & $1,15(0,71-1,85)$ & $1,87(1,04-3,37)$ & $1,59(0,83-3,04)$ \\
\hline
\end{tabular}

(continuação) 
Tabela 2 (continuação)

\begin{tabular}{|c|c|c|c|c|c|c|}
\hline \multirow[t]{4}{*}{ Variável } & \multirow{2}{*}{\multicolumn{2}{|c|}{$\begin{array}{c}\text { Modelo A * } \\
\text { Teste (sim/não) }\end{array}$}} & \multicolumn{4}{|c|}{ Modelo B ** } \\
\hline & & & \multirow{2}{*}{\multicolumn{2}{|c|}{$\begin{array}{c}\text { Teste solicitado } \\
\text { RC (IC95\%) }\end{array}$}} & \multirow{2}{*}{\multicolumn{2}{|c|}{$\begin{array}{c}\text { Teste espontâneo } \\
\text { RC (IC95\%) }\end{array}$}} \\
\hline & \multicolumn{2}{|c|}{ RC (IC95\%) } & & & & \\
\hline & Bruta & Ajustada *** & Bruta & Ajustada *** & Bruta & Ajustada *** \\
\hline Percepção de risco a & 0,381 \# & & 0,224 \# & & & \\
\hline \multicolumn{7}{|l|}{ HIV/DST } \\
\hline Muito elevada & 1,00 & - & 1,00 & - & 1,00 & - \\
\hline Elevada & $1,11(0,81-1,52)$ & - & $1,05(0,74-1,49)$ & - & $1,39(0,90-2,16)$ & - \\
\hline Moderada & $1,25(0,91-1,72)$ & - & $1,14(0,82-1,60)$ & - & $1,79(1,08-2,97)$ & - \\
\hline Baixa & $1,32(0,83-2,10)$ & - & $1,12(0,70-1,79)$ & - & $2,30(1,18-4,47)$ & - \\
\hline Muito baixa & $1,75(0,93-3,31)$ & - & $1,75(0,92-3,34)$ & - & $1,77(0,65-4,78)$ & - \\
\hline Ter tido pelo menos & $<0,001 \#$ & $<0,001 \#$ & $<0,001 \#$ & $<0,001 \#$ & & \\
\hline \multicolumn{7}{|l|}{ um filho biológico } \\
\hline Não & 1,00 & 1,00 & 1,00 & 1,00 & 1,00 & 1,00 \\
\hline Sim & $4,43(3,44-5.72)$ & $6,10(4,14-8,98)$ & $5,88(4,36-7,93)$ & $9,15(5,91-14,17)$ & $1,96(1,35-2,85)$ & $2,62(1,46-4,71)$ \\
\hline
\end{tabular}

IC95\%: intervalo de 95\% de confiança; RC: razão de chance.

* Modelo utilizando a variável realização de teste para HIV na forma dicotômica;

** Modelo utilizando a variável realização de teste para HIV na forma categórica nominal. Categoria de referência: nunca fez teste;

*** Ajustada para variáveis do mesmo nível e de níveis acima;

\# Valor de p referente ao teste de Wald.

\section{Tabela 3}

Razões de chance bruta e ajustada de realização de teste, teste solicitado e teste espontâneo para detecção do HIV, segundo escore de número de comportamentos sexuais de risco entre mulheres de 15-54 anos. Pelotas, Rio Grande do Sul, Brasil, 2012.

\begin{tabular}{|c|c|c|c|c|c|c|c|c|c|}
\hline \multirow[t]{4}{*}{ Variável } & \multirow{3}{*}{\multicolumn{3}{|c|}{$\begin{array}{c}\text { Modelo A * } \\
\text { Teste (sim/não) } \\
\text { RC (IC95\%) }\end{array}$}} & \multicolumn{6}{|c|}{ Modelo B ** } \\
\hline & & & & \multirow{2}{*}{\multicolumn{3}{|c|}{$\begin{array}{l}\text { Teste solicitado } \\
\text { RC (IC95\%) }\end{array}$}} & \multirow{2}{*}{\multicolumn{3}{|c|}{$\begin{array}{c}\text { Teste espontâneo } \\
\text { RC (IC95\%) }\end{array}$}} \\
\hline & & & & & & & & & \\
\hline & Bruta & Ajustada *** & Ajustada \# & Bruta & Ajustada *** & Ajustada \# & Bruta & Ajustada *** & Ajustada \# \\
\hline $\begin{array}{l}\text { Escore de } \\
\text { número } \\
\text { de compor- } \\
\text { tamentos } \\
\text { sexuais }\end{array}$ & 0,002 \#\# & 0,001 \#\# & 0,023 \#\# & 0,014 \#\# & 0,010 \#\# & 0,117 \#\# & & & \\
\hline Nenhum & 1,00 & 1,00 & 1,00 & 1,00 & 1,00 & 1,00 & 1,00 & 1,00 & 1,00 \\
\hline 1 & $\begin{array}{c}1,19 \\
(0,75-1,88)\end{array}$ & $\begin{array}{c}1,22 \\
(0,75-1,97)\end{array}$ & $\begin{array}{c}1,16 \\
(0,68-1,96)\end{array}$ & $\begin{array}{c}1,23 \\
(0,75-2,01)\end{array}$ & $\begin{array}{c}1,24 \\
(0,74-2,09)\end{array}$ & $\begin{array}{c}1,19 \\
(0,67-2,11)\end{array}$ & $\begin{array}{c}1,06 \\
(0,56-2,01)\end{array}$ & $\begin{array}{c}1,14 \\
(0,57-2,25)\end{array}$ & $\begin{array}{c}1,08 \\
(0,53-2,18)\end{array}$ \\
\hline 2 & $\begin{array}{c}1,69 \\
(1,12-2,54)\end{array}$ & $\begin{array}{c}1,67 \\
(1,09-2,56)\end{array}$ & $\begin{array}{c}1,51 \\
(0,94-2,42)\end{array}$ & $\begin{array}{c}1,79 \\
(1,16-2,76)\end{array}$ & $\begin{array}{c}1,69 \\
(1,06-2,68)\end{array}$ & $\begin{array}{c}1,51 \\
(0,90-2,51)\end{array}$ & $\begin{array}{c}1,37 \\
(0,73-2,60)\end{array}$ & $\begin{array}{c}1,63 \\
(0,84-3,18)\end{array}$ & $\begin{array}{c}1,54 \\
(0,78-3,03)\end{array}$ \\
\hline 3 ou mais & $\begin{array}{c}2,26 \\
(1,31-3,91)\end{array}$ & $\begin{array}{c}2,46 \\
(1,40-4,29)\end{array}$ & $\begin{array}{c}1,96 \\
(1,10-3,49)\end{array}$ & $\begin{array}{c}2,33 \\
(1,28-4,25)\end{array}$ & $\begin{array}{c}2,48 \\
(1,33-4,62)\end{array}$ & $\begin{array}{c}1,90 \\
(0,99-3,63)\end{array}$ & $\begin{array}{c}2,03 \\
(0,99-4,18)\end{array}$ & $\begin{array}{c}2,42 \\
(1,13-5,20)\end{array}$ & $\begin{array}{c}2,13 \\
(0,99-4,56)\end{array}$ \\
\hline
\end{tabular}

IC95\%: intervalo de 95\% de confiança; RC: razão de chance.

* Modelo utilizando a variável realização de teste para HIV na forma dicotômica;

** Modelo utilizando a variável realização de teste para HIV na forma categórica nominal, tendo como categoria de referência "nunca fez teste";

*** Ajustada para idade, renda, escolaridade e situação conjugal;

\# Ajustada para idade, renda, escolaridade, situação conjugal e ter tido pelo menos um filho biológico;

\#\# Valor de $\mathrm{p}$ referente ao teste de Wald. 
apresentaram 1,39 vez mais chance de realização de teste, quando comparado a mulheres que viviam sem companheiro. Prática de sexo anal, primeira relação sexual precoce e ter tido pelo menos um filho biológico também se mostraram associadas à realização de teste. Mulheres que praticaram sexo anal iniciaram a vida sexual em idade precoce e tiveram pelo menos um filho biológico apresentaram chance 2,5, 1,40 e 6,10 vezes maior de realização de teste para HIV, respectivamente. Os resultados das análises bruta e ajustada para o desfecho A são apresentados na Tabela 2.

A Tabela 3 apresenta as razões de chances de realização de teste para HIV e escore de comportamentos sexuais de risco. A análise bruta evidenciou aumento na chance de teste, conforme aumentou o número de comportamentos sexuais de risco relatados. Após ajuste para variáveis demográficas e socioeconômicas, o resultado se manteve semelhante. Mulheres que relataram dois comportamentos sexuais de risco apresentaram 1,67 vez mais chance de teste, enquanto a mesma chance foi 2,46 vezes maior para aquelas que relataram três ou mais comportamentos. Quando procedido ajuste adicional para a variável parição, apenas a presença de três ou mais comportamentos se manteve associada com realização de teste $(\mathrm{RC}=1,96)$.

\section{- Análise do desfecho B}

A análise ajustada identificou associação teste solicitado e as variáveis idade, renda, situação conjugal, uso de preservativo e primeira relação sexual precoce; e estiveram associadas a teste espontâneo as variáveis idade, prática de sexo anal, primeira relação precoce e parição. A idade apresentou forte associação, tanto com teste solicitado, quanto com teste espontâneo, com maiores chances de teste para as mulheres entre 30-39 anos ( $\mathrm{RC}=15,12$ e $\mathrm{RC}=10,70$, respectivamente). Mulheres pertencentes ao quintil inferior de renda apresentaram 1,79 vez mais chance de teste solicitado, mas não houve associação desta variável com teste espontâneo. Mulheres com companheiro apresentaram 70\% mais chance de teste solicitado, porém essa associação não foi observada para teste espontâneo. Mulheres que não utilizaram preservativo na última relação apresentaram 39\% mais chance de teste solicitado, em comparação com aquelas que usaram. Não existiu diferença entre proporções de teste espontâneo segundo essa variável. Não foi evidenciado efeito da prática de sexo anal sobre teste solicitado, apenas sobre teste espontâneo. Mulheres que praticaram sexo anal apresentaram 3,57 vezes mais chance de teste espontâneo em comparação com aquelas que não praticaram. Ter tido pelo menos um filho biológico esteve associado tanto a teste solicitado quanto a espontâneo. A chance de teste solicitado foi 9,15 vezes maior entre aquelas que tiveram pelo menos um filho biológico, enquanto a chance de teste espontâneo entre elas foi de 2,62. Os resultados das análises bruta e ajustada para o desfecho B são apresentados na Tabela 2.

A Tabela 3 apresenta as razões de chances de realização de teste para HIV e escore de comportamentos sexuais de riso. A análise evidenciou aumento na chance de teste solicitado, conforme aumento do número de comportamentos sexuais de risco relatados. Mulheres que relataram dois comportamentos sexuais de risco apresentaram chance $69 \%$ maior de teste solicitado, enquanto aquelas que relataram três ou mais comportamentos apresentaram chance 148\% maior. Quando procedido ajuste adicional para a variável parição, as associações perderam a significância estatística.

Com relação à realização de teste espontâneo, a análise ajustada evidenciou efeito positivo do relato de três ou mais comportamentos sobre realização de teste espontâneo $(\mathrm{RC}=2,42)$. Após ajuste para a variável parição, a associação entre teste solicitado e presença de três ou mais comportamentos sexuais de risco perdeu a significância estatística.

Com relação à avaliação de qualidade do ajuste do modelo, os valores dos parâmetros utilizados foram os seguintes: o modelo multinomial utilizado apresentou AIC $=1847$ e BIC $=1966$ e o modelo multinomial com todas as variáveis apresentou $\mathrm{AIC}=1824$ e BIC $=2002$. Já o modelo logístico apresentou $\mathrm{AIC}=1199$ e $\mathrm{BIC}=1259$ e o modelo com todas as variáveis, $\mathrm{AIC}=1147$ e $\mathrm{BIC}=1236$. Esses resultados indicam que os modelos foram adequados, tendo em vista que, apesar de um menor número de variáveis, as medidas de qualidade de ajuste se mantiveram semelhantes. 


\section{Discussão}

Diversos estudos de base populacional realizados em diferentes países investigaram a prevalência de realização de teste para HIV e fatores associados 9,10,11,12,13,14,15,16,17,18,19,20,21. É importante salientar que existe, na literatura, uma ampla variação na definição operacional de teste solicitado e teste espontâneo, o que prejudica a comparação dos resultados e pode explicar a discrepância entre os resultados deste estudo e outros achados da literatura.

A prevalência de realização de teste para HIV alguma vez durante a vida, referida por $66 \%$ das entrevistadas, é superior aos resultados encontrados em outros estudos de base populacional, os quais variaram entre $27 \%$ e $44 \%$ 9,10,11,12,13,14,15,16,17. A prevalência de realização de teste para HIV solicitado foi de $52 \%$, superior às relatadas por outros estudos em que a prevalência variou entre 27 e $45 \%$ 10,11,13,14. Já a prevalência de realização de teste para HIV espontâneo foi de $14 \%$, sendo ela intermediária, se comparada com os achados da literatura, que mostraram prevalências entre $3 \mathrm{e}$ $33 \%$ 10,13. O motivo para realização de teste mais frequente apontado foi rastreamento pré-natal, seguido de doação de sangue, resultado consistente com os achados de McGarrigle et al. 11, Dandona et al. 12, Sambisa et al. 13 e de la Fuente et al. 14.

A idade mostrou-se associada a teste espontâneo e a teste solicitado, achado consistente com a literatura que, de maneira geral, aponta a faixa 20-40 anos como a mais comumente testada 9,11,16,18,19. Tal resultado, provavelmente, se deve ao fato de essa ser a faixa etária em que ocorre a maioria das gestações e, portanto, há maior solicitação de teste pelos profissionais de saúde. A associação foi verificada também quando considerado apenas o teste espontâneo. É possível que mulheres nessa faixa etária sejam sexualmente mais ativas e tenham crescido na "era AIDS", o que as leva a maior consciência da necessidade de realização do teste.

A situação conjugal esteve associada à realização de teste para HIV solicitado, resultado inconsistente com a literatura 9,11,12,14,15,16,17,20. Maior chance de teste solicitado entre mulheres casadas ou com companheiro pode ser reflexo do fato de ter tido pelo menos um filho biológico, uma vez que $85 \%$ das mulheres com companheiro, nesta amostra, declararam ter tido pelo menos um filho biológico, enquanto entre as não casadas o percentual foi de $47 \%$ (dados não apresentados nos resultados).

Primeira relação sexual precoce também esteve associada à realização de teste. Esse resultado é compatível com o encontrado por outro estudo brasileiro conduzido por França Junior et al. 9. É possível que a mulher que tenha tido a primeira relação sexual precocemente engravide mais cedo, o que explicaria a maior chance de teste solicitado. A associação entre primeira relação sexual precoce e teste espontâneo também foi identificada. A mulher que inicia a vida sexual mais cedo, provavelmente, tem mais parceiros ao longo da vida, o que aumenta a probabilidade de realização de teste, conforme documentado na literatura 9,10,11,12,14,19. Tal relação não foi verificada neste estudo, entretanto é preciso considerar que o período recordatório utilizado para múltiplos parceiros sexuais (3 meses) é curto, o que pode explicar a discrepância com os resultados encontrados por outros estudos. A não utilização de preservativo também esteve associada à realização de teste solicitado. Isso também pode ser um reflexo da parição, uma vez que o uso de preservativo é um importante método de prevenção da gravidez, e análises adicionais não apresentadas na seção de resultados evidenciaram associação entre não uso de preservativo e parição ( $\mathrm{RC}=2,03$; IC95\%: 1,58-2,61). Outra hipótese é que profissionais de saúde percebem o risco a que a mulher está exposta e, portanto, solicitam mais o teste. A ausência de associação entre não uso de preservativo e teste espontâneo pode indicar que a mulher não tem adequada percepção sobre o seu risco de adquirir HIV, o que já foi demonstrado em estudo conduzido com a mesma população 21.

Vale destacar a associação de considerável magnitude entre realização de teste espontâneo e prática de sexo anal, que se manteve mesmo após ajuste para variáveis sociodemográficas e para outros comportamentos sexuais. Há maior probabilidade de transmissão do HIV por ato, durante a relação sexual anal, quando comparada a relação sexual vaginal ou oral 22. É possível que tal resultado esteja ligado ao conhecimento por parte das mulheres dessa maior probabilidade de transmissão, fato que levaria aquelas que o praticam a se perceberem sob risco de infecção e, consequentemente, submeterem-se voluntariamente ao teste.

Tendo em vista maior prevalência de realização de teste solicitado, a principal motivação para realização (pré-natal) e maior chance de realização de teste para HIV entre mulheres que tiveram pelo 
menos um filho biológico, fica evidente que a realização de teste está fortemente relacionada com a atenção pré-natal, panorama já identificado no Brasil por França Junior et al. ${ }^{9}$. Como demonstrado por Veloso et al. 23 e Goldani et al. 24, a prevalência de realização do teste durante a gestação é alta. Apesar de o teste durante a gestação ser facultativo, é comum a gestante não ser informada sobre seu direito em recusá-lo ou receber a informação de que a realização é obrigatória. Algumas mulheres, embora saibam sobre o caráter facultativo do teste, diante dos profissionais de saúde, podem sentirse constrangidas em negar a realização 23,24. Esses fatores, aliados à consciência da gestante sobre a importância do diagnóstico precoce para prevenção da transmissão vertical, contribuem para que a prevalência de testagem durante o pré-natal seja alta, quando comparada à prevalência de teste espontâneo, que encontra no estigma associado ao HIV/AIDS uma barreira.

A alta prevalência de teste motivado por rastreamento pré-natal e a baixa prevalência de teste espontâneo indicam que uma importante parcela da população de mulheres que não teve filho(s) biológico(s) ou não recebe a assistência pré-natal, provavelmente, não realiza teste de HIV. Mesmo em menor magnitude, a parição está relacionada também à realização de teste espontâneo, o que pode indicar que a mulher que tem filho(s) é mais cuidadosa com sua saúde, seja por preocupação com a capacidade de cuidado com a criança ou pelo fato de a mulher ter mais contato com o serviço de saúde, o que levaria a maior consciência sobre sua própria saúde.

A procura pelo teste de forma espontânea é mais complexa. O estigma e a discriminação associados ao HIV/AIDS podem reduzir ou dificultar a realização do teste por causa do medo do resultado e a busca por tratamento, caso positivo 25,26 . Tal fato, talvez contribua para que o número de mulheres que busca espontaneamente pelo teste seja baixo, apesar dos esforços do governo brasileiro com iniciativas como o programa Fique Sabendo, lançado em 2003, que estimula a realização do teste rápido. Atualmente, outras ações vêm sendo desenvolvidas para estimular a testagem. Entre elas, o programa Quero Saber, que utiliza unidades móveis (trailers) para levar testes a locais de maior frequência de populações vulneráveis, e o Viva Melhor Sabendo, realizado em parceria entre o Ministério da Saúde e organizações não governamentais. Espera-se que essas ações contribuam para o aumento na realização de testes espontâneos no país, nos próximos anos 27.

A análise entre realização de teste para HIV e o escore de comportamento sexual, mesmo após ajuste para parição, evidenciou aumento progressivo na chance de teste solicitado, com maiores chances entre mulheres que apresentam dois comportamentos e três comportamentos ou mais. Não foi evidenciada associação entre escore de comportamentos e realização de teste espontâneo, o que pode indicar que o profissional de saúde percebe o risco a que a mulher está exposta de maneira mais adequada do que a própria. Estudo conduzido por Silveira et al. 21, com a mesma população, evidenciou que as mulheres não percebem adequadamente seu nível de risco.

Este estudo possui limitações. A questão sobre motivação para realização de teste de HIV permite conhecer apenas o principal motivo para realização do último teste, entretanto algumas mulheres podem ter realizado o teste por mais de um motivo. É importante mencionar a dificuldade em relatar experiências íntimas. Questionário autoaplicado e confidencial foi utilizado para minimizar problemas nos relatos, mesmo assim pode ter havido tendência a fornecer respostas socialmente aceitas.

Este parece ser o primeiro estudo brasileiro de base populacional a avaliar, separadamente, fatores associados à realização de teste solicitado e teste espontâneo, incluindo variáveis de comportamento sexual. O complexo processo de amostragem e o baixo percentual de perdas e recusas minimizam a possibilidade de viés de seleção e asseguram a representatividade da amostra, garantindo a validade interna do estudo. Ainda alguns resultados deste estudo vão ao encontro de achados de publicação nacionalmente representativa 9 , o que leva a crer que eles podem ser extrapolados para a população de mulheres de outras cidades de médio porte brasileiras e poderia evidenciar a validade externa deste estudo.

Este estudo mostrou que o teste para HIV, independentemente do motivo, foi realizado por aproximadamente dois terços das mulheres. Teste solicitado foi realizado por cerca de metade das mulheres e está associado à idade, renda, situação conjugal, não uso de preservativo, primeira relação sexual precoce, ao fato de a mulher ter tido pelo menos um filho biológico e presença de dois ou mais comportamentos sexuais de risco. Já a frequência de realização de teste para HIV espontâneo é baixa e está associado à idade, prática de sexo anal, primeira relação precoce, percepção de risco e ao fato de a mulher ter tido pelo menos um filho biológico. 
Pode-se concluir que a prevalência de realização de teste espontâneo é baixa e que a realização de teste para HIV entre mulheres está associada, sobretudo, à rotina pré-natal. Apesar dos esforços por parte do governo brasileiro em estimular a realização de teste pelo programa Fique Sabendo, a testagem espontânea, independentemente do risco de infecção, está aquém do ideal. É necessário, portanto, ampliar os esforços de conscientização, não apenas das mulheres, mas da população como um todo, sobre a importância da descoberta do soro status, pois sua descoberta precoce permite, com a administração adequada de TARV - de acesso gratuito e universal -, não só um aumento da sobrevida do portador, como também a diminuição da possibilidade de transmissão do vírus.

\section{Colaboradores}

M. A. Mesenburg participou de todas as etapas do estudo, incluindo concepção e projeto, análise e interpretação dos dados, redação do artigo e aprovação da versão a ser publicada. F. C. Wehrmeister participou da análise e interpretação dos dados, realizou revisão crítica relevante do conteúdo intelectual e aprovou a versão a ser publicada. M. F. Silveira participou da concepção, projeto e interpretação dos dados, realizou revisão crítica relevante do conteúdo intelectual e aprovou a versão a ser publicada.

\section{Agradecimentos}

Agradecimento à Coordenação de Aperfeiçoamento de Pessoal de Nível Superior (Capes) pelo apoio financeiro.

\section{Referências}

1. Joint United Nations Programme on HIV/ AIDS. The gap report. Geneva: Joint United Nations Programme on HIV/AIDS; 2014.

2. Departamento de DST, AIDS e Hepatites Virais, Secretaria de Vigilância em Saúde, Ministério da Saúde. Boletim Epidemiológico - HIV e Aids 2014; Ano III, no 1.

3. Marks G, Crepaz N, Senterfitt JW, Janssen RS. Meta-analysis of high-risk sexual behavior in persons aware and unaware they are infected with HIV in the United States: implications for HIV prevention programs. J Acquir Immune Defic Syndr 2005; 39:446-53

4. Cohen MS, Chen YQ, McCauley M, Gamble T, Hosseinipour MC, Kumarasamy N, et al. Prevention of HIV-1 infection with early antiretroviral therapy. N Engl J Med 2011; 365:493505.

5. Bastos FI, Szwarcwald CL. AIDS e pauperização: principais conceitos e evidências empíricas. Cad Saúde Pública 2000; 16 Suppl 1:65-76.

6. Joint United Nations Programme on HIV/ AIDS. Global report: UNAIDS report on the global AIDS epidemic 2010. Geneva: Joint United Nations Programme on HIV/AIDS; 2010.

7. Ministério da Saúde. Plano integrado de enfrentamento da feminização da epidemia da Aids e outras DST. Brasília: Ministério da Saúde; 2009. 
8. Barros AJD, Menezes AMB, Santos IS, Assunção MCF, Gigante D, Fassa AG, et al. O mestrado do Programa de Pós-graduação em Epidemiologia da UFPel baseado em consórcio de pesquisa: uma experiência inovadora. Rev Bras Epidemiol 2008; 11:133-44.

9. França Junior I, Calazans G, Zucchi EM. Mudanças no âmbito da testagem anti-HIV no Brasil entre 1998 e 2005. Rev Saúde Pública 2008; 42 Suppl 1:84-97.

10. Renzi C, Zantedeschi E, Signorelli C, Osborn JF. Factors associated with HIV testing: results from an Italian General Population Survey. Prev Med 2001; 32:40-8.

11. McGarrigle CA, Mercer CH, Fenton KA, Copas AJ, Wellings K, Erens B, et al. Investigating the relationship between HIV testing and risk behaviour in Britain: National Survey of Sexual Attitudes and Lifestyles 2000. AIDS 2005; 19:77-84.

12. Dandona R, Kumar SG, Kumar GA, Lakshmi $\mathrm{V}$, Dandona L. HIV testing among adults in a high prevalence district in India. Natl Med J India 2009; 22:289-93.

13. Sambisa W, Curtis S, Mishra V. AIDS stigma as an obstacle to uptake of HIV testing: evidence from a Zimbabwean national populationbased survey. AIDS Care 2010; 22:170-86.

14. de la Fuente L, Suarez M, Belza MJ, Vallejo F, Garcia M, Alvarez R, et al. Human immunodeficiency virus testing uptake and risk behaviours in Spain. J Epidemiol Community Health 2009; 63:552-8.

15. Huchko MJ, Montandon M, Nguti R, Bukusi EA, Cohen CR. The association of HIV counseling and testing with HIV risk behaviors in a random population-based survey in Kisumu, Kenya. AIDS Behav 2011; 15:718-24.

16. Agha S. Factors associated with HIV testing and condom use in Mozambique: implications for programs. Reprod Health 2012; 9:20.

17. Cherutich P, Kaiser R, Galbraith J, Williamson J, Shiraishi RW, Ngare C, et al. Lack of knowledge of HIV status a major barrier to HIV prevention, care and treatment efforts in Kenya: results from a nationally representative study. PLoS One 2012; 7:e36797.

18. Lemcke A, Kjoller M, Ekholm O, Smith E. HIV testing in the Danish population: a national representative survey, 2000. Scand J Public Health 2007; 35:631-9.
19. Sonnenberg P, Clifton S, Beddows S, Field N, Soldan K, Tanton C, et al. Prevalence, risk factors, and uptake of interventions for sexually transmitted infections in Britain: findings from the National Surveys of Sexual Attitudes and Lifestyles (Natsal). Lancet 2013; 382: 1795-806.

20. Venkatesh KK, Madiba P, De Bruyn G, Lurie MN, Coates TJ, Gray GE. Who gets tested for HIV in a South African urban township? Implications for test and treat and gender-based prevention interventions. J Acquir Immune Defic Syndr 2011; 56:151-65.

21. Silveira MF, Béria JU, Horta BL, Tomasi E. Autopercepção de vulnerabilidade às doenças sexualmente transmissíveis e Aids em mulheres. Rev Saúde Pública 2002; 36:670-7.

22. Public Health Agency of Canada. HIV transmisson risk: a summary of the evidence. Ottawa: Public Health Agency of Canada; 2012.

23. Veloso VG, Portela MC, Vasconcellos MT, Matzenbacher LA, Vasconcelos AL, Grinsztejn $\mathrm{B}$, et al. HIV testing among pregnant women in Brazil: rates and predictors. Rev Saúde Pública 2008; 42:859-67.

24. Goldani MZ, Giugliani ER, Scanlon T, Rosa H, Castilhos K, Feldens L, et al. Voluntary HIV counseling and testing during prenatal care in Brazil. Rev Saúde Pública 2003; 37:552-8.

25. Herek GM, Capitanio JP, Widaman KF. HIVrelated stigma and knowledge in the United States: prevalence and trends, 1991-1999. Am J Public Health 2002; 92:371-7.

26. Herek GM, Capitanio JP, Widaman KF. Stigma, social risk, and health policy: public attitudes toward HIV surveillance policies and the social construction of illness. Health Psychol 2003; 22:533-40.

27. Departamento de DST, AIDS e Hepatites Virais, Secretaria de Vigilância em Saúde, Ministério da Saúde. Saúde realiza mobilização nacional de prevenção e testagem de sífilis, HIV e hepatites virais. http://www.aids.gov.br/noti cia/2012/saude-realiza-mobilizacao-nacionalde-prevencao-e-testagem-de-sifilis-hiv-e-he patites-virais (acessado em 10/Ago/2015). 


\section{Abstract}

This was a cross-sectional population-based study that aimed to describe the prevalence of HIV testing and associated factors in women in Pelotas, Rio Grande do Sul State, Brazil. A total of 1,222 women were interviewed. We estimated the overall testing prevalence (yes/no) and prevalence disaggregated by testing ordered by a health professional versus voluntary testing. Test prevalence was 66.1\% (95\% CI: 63.4-68.8): 52.4\% for testing ordered by a health professional (95\%CI: 49.6-55.2) and $13.6 \%$ for spontaneous testing (95\%CI: 11.615.5). The principal reason for testing was prenatal screening (52\%). Age, age at sexual initiation, and having children were associated statistically with both voluntary and health professional-initiated testing. Sexual risk score, conjugal status, and condom use were only associated statistically with testing ordered by a health professional, while history of anal sex was only associated with spontaneous testing. The results indicate that HIV testing is closely related to prenatal care and that risk perception by the attending health professional appears to be more accurate than the patient's own perception.

HIV; AIDS Serodiagnosis; Women

\section{Resumen}

Se realizó un estudio transversal de base poblacional, con el objetivo de describir la prevalencia, motivos y factores asociados para la realización del test para VIH entre mujeres de Pelotas, Sur de Brasil. Se entrevistaron a 1.222 mujeres. Se estimó la prevalencia de test (si/no), test solicitado y test voluntario. La prevalencia de test fue de un 66,1\% (IC95\%: 63,4-68,8), test solicitado un 52,4\% (IC95\%: 49,6-55,2) y test espontáneo 13,6\% (IC95\%: 11,6-15,5). El principal motivo para la realización del test fue el rastreo pre-natal (52\%). Edad, edad de la primera relación y tener hijos se mostraron asociados a la realización de test espontáneo y solicitado. El marcador de comportamientos sexuales de riesgo, situación conyugal y uso de preservativo se mostraron asociados solamente al test solicitado y práctica de sexo anal solamente a test espontáneo. Los resultados indican que el test para VIH parece estar fuertemente relacionado con la asistencia pre-natal y que la percepción de riesgo por parte de los profesionales de salud parece ser más adecuada que la percepción de la propia mujer.

VIH; Serodiagnóstico del SIDA; Mujeres
Recebido em 11/Mai/2015

Versão final reapresentada em 28/Jul/2015

Aprovado em 13/Dez/2016 